\title{
COVID-19 apps for the ObGyn health care provider: An update
}

\author{
Ten apps to help clinicians stay informed about COVID-19 and evolving \\ clinical decision making
}

Lillian C. Chen, MD, MPH, and Katherine T. Chen, MD, MPH

IN THIS

ARTICLE

Details on recommended apps

page 47
$\mathrm{M}$ ore than one year after COVID-19 was declared a worldwide pandemic by the World Health Organization on March 11, 2020, the disease continues to persist, infecting more than 110 million individuals to date globally. ${ }^{1}$ As new information emerges about the coronavirus, the literature on diagnosis and management also has grown exponentially over the last year, including specific guidance for obstetric populations. With abundant information available to health care providers, COVID-19 mobile apps have the advantage of summarizing and presenting information in an organized and easily accessible manner. ${ }^{2}$

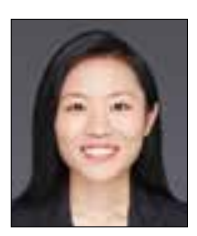

Dr. Lillian Chen is a first-year resident in the Department of Obstetrics, Gynecology, and Reproductive Science, Icahn School of Medicine at Mount Sinai, New York, New York.

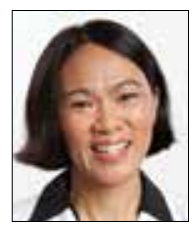

Dr. Katherine Chen is Professor of Obstetrics, Gynecology, and Reproductive Science and Medical Education, Vice-Chair of Ob-Gyn Education for the Mount Sinai Health System, Icahn School of Medicine at Mount Sinai, New York. She is an OBG MANAGEMENT Contributing Editor.

Dr. Katherine Chen reports being an advisory board member and receiving royalties from UpToDate, Inc. Dr. Lillian Chen reports no financial relationships relevant to this article.

doi: 10.12788/obgm.0088
This updated review expands on a previous article by Bogaert and Chen at the start of the COVID-19 pandemic. ${ }^{3}$ Using the same methodology, in March 2021 we searched the Apple iTunes and Google Play stores using the term "COVID." The search yielded 230 unique applications available for download. We excluded apps that were primarily developed as geographic area-specific case trackers or personal symptom trackers (193), those that provide telemedicine services (7), and nonmedical apps or ones published in a language other than English (20).

Here, we focus on the 3 mobile apps previously discussed (CDC, My Osler, and Relief Central) and 7 additional apps (TABLE). Most summarize information on the prevention, diagnosis, and treatment of coronavirus, and several also provide information on the COVID-19 vaccine. One app (COVID-19 Resource for Midwives) is specifically designed for obstetric providers, and 4 others (CDC, COVID-19 Protocols, Medscape, and WHO Academy) contain information on specific guidance for obstetric and gynecologic patient populations.

Each app was evaluated based on a condensed version of the APPLICATIONS scoring system, APPLI (comprehensiveness, price, platform, literature used, and special features). ${ }^{4}$

We hope that these mobile apps will assist the ObGyn health care provider in continuing to care for patients during this pandemic. 
TABLE Recommended COVID-19 apps

\begin{tabular}{|c|c|c|c|c|c|}
\hline App & Comprehensiveness & Price & Platform & $\begin{array}{l}\text { Literature } \\
\text { used }\end{array}$ & $\begin{array}{l}\text { Important special } \\
\text { features }\end{array}$ \\
\hline $\begin{array}{l}\text { iTunes: https://apps.apple.com/ } \\
\text { us/app/cdc/id487847188 } \\
\text { Google Play: https:// } \\
\text { play.google.com/store/ } \\
\text { apps/details?id=gov.cdc. } \\
\text { general\&hl=en_US\&gl=US }\end{array}$ & $\begin{array}{l}\text { - Clinical decision mak- } \\
\text { ing (clinical treatment } \\
\text { guidelines) } \\
\text { - Reference and } \\
\text { information gathering } \\
\text { (medical literature, } \\
\text { medical news) }\end{array}$ & Free & $\begin{array}{l}\text { iTunes and } \\
\text { Google Play }\end{array}$ & $\begin{array}{l}\text { CDC web- } \\
\text { site, scientific } \\
\text { journals }\end{array}$ & $\begin{array}{l}\text { - Section on care for } \\
\text { pregnant patients } \\
\text { - Vaccine updates } \\
\text { - Guidelines for } \\
\text { workplace safety } \\
\text { and infection } \\
\text { control } \\
\text { - Videos on testing, } \\
\text { vaccination, and } \\
\text { symptom review } \\
\text { targeted toward } \\
\text { health care provid- } \\
\text { ers and patients }\end{array}$ \\
\hline $\begin{array}{l}\text { CoviD-19: Response } \\
\text { (United Nations) } \\
\text { iTunes: https://apps.apple.com/ } \\
\text { de/app/covid-19-response/ } \\
\text { id1513391764?I=en } \\
\text { Google Play: https:// } \\
\text { play.google.com/store/ } \\
\text { apps/details?id=org. } \\
\text { un.corona\&hl=en_US\&gl=US }\end{array}$ & $\begin{array}{l}\text { - Clinical decision mak- } \\
\text { ing (clinical decision } \\
\text { support systems) } \\
\text { - Reference and } \\
\text { information gathering } \\
\text { (medical literature, } \\
\text { medical news) }\end{array}$ & Free & $\begin{array}{l}\text { iTunes and } \\
\text { Google Play }\end{array}$ & $\begin{array}{l}\text { UN, WHO, } \\
\text { PAHO, scien- } \\
\text { tific journals }\end{array}$ & $\begin{array}{l}\text { Dedicated } \\
\text { resources for pa- } \\
\text { tients, health care } \\
\text { workers } \\
\text { - Overview of } \\
\text { global COVID-19 } \\
\text { goals regard- } \\
\text { ing health care } \\
\text { policy, economics, } \\
\text { regional pandemic } \\
\text { overviews } \\
\text { - Resources on } \\
\text { well-being and } \\
\text { domestic violence }\end{array}$ \\
\hline $\begin{array}{l}\begin{array}{c}\text { BRIOHAM } \\
\text { HEALTH }\end{array} \\
\text { COVID-19 Protocols (Brigham } \\
\text { and Women's Hospital) } \\
\text { iTunes: https://apps.apple. } \\
\text { com/us/app/covid-protocols/ } \\
\text { id1514563997 }\end{array}$ & $\begin{array}{l}\text { - Clinical decision mak- } \\
\text { ing (clinical treatment } \\
\text { guidelines, disease } \\
\text { diagnosis aids) } \\
\text { - Reference and } \\
\text { information gathering } \\
\text { (medical literature) }\end{array}$ & Free & iTunes & $\begin{array}{l}\text { CDC, scientific } \\
\text { journals }\end{array}$ & $\begin{array}{l}\text { - Section on care of } \\
\text { pregnant patients } \\
\text { - Vaccine updates } \\
\text { - Guides for patient } \\
\text { management and } \\
\text { infection control } \\
\text { in different health } \\
\text { care settings }\end{array}$ \\
\hline $\begin{array}{l}\text { AON/ } \\
\text { CoviD-19 Resource for } \\
\text { Midwives (Association of } \\
\text { Ontario Midwives) } \\
\text { iTunes: https://apps.apple.com/ } \\
\text { us/app/covid-19-resource-for- } \\
\text { midwives/id1510033043 }\end{array}$ & $\begin{array}{l}\text { - Clinical decision mak- } \\
\text { ing (clinical treatment } \\
\text { guidelines, laboratory } \\
\text { test ordering) } \\
\text { - Reference and } \\
\text { information gathering } \\
\text { (medical literature) }\end{array}$ & Free & iTunes & $\begin{array}{l}\text { Provincial } \\
\text { Council for } \\
\text { Maternal and } \\
\text { Child Health, } \\
\text { scientific } \\
\text { journals }\end{array}$ & $\begin{array}{l}\text { Guides for screen- } \\
\text { ing, antepartum, } \\
\text { intrapartum, } \\
\text { and postpartum } \\
\text { management of } \\
\text { patients }\end{array}$ \\
\hline
\end{tabular}


TABLE Recommended COVID-19 apps (continued)

\begin{tabular}{|c|c|c|c|c|c|}
\hline App & Comprehensiveness & Price & Platform & $\begin{array}{c}\text { Literature } \\
\text { used }\end{array}$ & $\begin{array}{c}\text { Important special } \\
\text { features }\end{array}$ \\
\hline $\begin{array}{l}\text { Epocrates } \\
\text { iTunes: https://apps } \\
\text {.apple.com/us/app/ } \\
\text { epocrates/id281935788 } \\
\text { Google Play: https:// } \\
\text { play.google.com/store/ } \\
\text { apps/details?id=com. } \\
\text { epocrates\&hl=en_US\&gl=US }\end{array}$ & $\begin{array}{l}\text { Clinical decision mak- } \\
\text { ing (clinical treatment } \\
\text { guidelines) } \\
\text { - Reference and } \\
\text { information gathering } \\
\text { (medical literature) }\end{array}$ & $\begin{array}{l}\text { Free basic } \\
\text { information, } \\
\$ 16.99 \text { for } \\
\text { premium } \\
\text { subscription }\end{array}$ & $\begin{array}{l}\text { iTunes and } \\
\text { Google Play }\end{array}$ & $\begin{array}{l}\text { CDC, WHO, } \\
\text { scientific } \\
\text { journals }\end{array}$ & $\begin{array}{l}\text { Treatment guide- } \\
\text { lines stratified by } \\
\text { age and disease } \\
\text { status } \\
\text { - Drug and vaccine } \\
\text { trial updates } \\
\text { - Billing and coding } \\
\text { guidelines for } \\
\text { providers }\end{array}$ \\
\hline $\begin{array}{l}\text { First Responder COVID-19 } \\
\text { Guide (Stanford Medicine) } \\
\text { iTunes: https://apps.apple.com/ } \\
\text { us/app/first-responder-covid- } \\
\text { 19-guide/id1505677599 }\end{array}$ & $\begin{array}{l}\text { - Clinical decision mak- } \\
\text { ing (clinical treatment } \\
\text { guidelines) } \\
\text { - Reference and } \\
\text { information gathering } \\
\text { (medical literature) }\end{array}$ & Free & iTunes & $\begin{array}{l}\text { Stanford } \\
\text { Medicine, } \\
\text { CDC, scientific } \\
\text { journals }\end{array}$ & $\begin{array}{l}\text { - Guidance on test- } \\
\text { ing and infection } \\
\text { control } \\
\text { - Information } \\
\text { regarding current } \\
\text { clinical research } \\
\text { and clinical trials }\end{array}$ \\
\hline $\begin{array}{l}\text { Medscape } \\
\text { Medscape } \\
\text { iTunes: https://apps.apple. } \\
\text { com/us/app/medscape/ } \\
\text { id321367289 } \\
\text { Google Play: https://play. } \\
\text { google.com/store/apps/ } \\
\text { details?id=com.medscape. } \\
\text { android\&hl=en_US\&gl=US }\end{array}$ & $\begin{array}{l}\text { - Clinical decision mak- } \\
\text { ing (clinical treatment } \\
\text { guidelines) } \\
\text { - Reference and } \\
\text { information gathering } \\
\text { (medical literature, } \\
\text { medical news) }\end{array}$ & Free & $\begin{array}{l}\text { iTunes and } \\
\text { Google Play }\end{array}$ & $\begin{array}{l}\text { CDC, WHO, } \\
\text { scientific } \\
\text { journals }\end{array}$ & $\begin{array}{l}\text { Video on diagno- } \\
\text { sis/management } \\
\text { of COVID-19 in } \\
\text { pregnancy } \\
\text { - Multimedia re- } \\
\text { sources (articles, } \\
\text { videos) } \\
\text { - Updates on vac- } \\
\text { cine and drug } \\
\text { trials } \\
\text { - Guidance for spe- } \\
\text { cific professions } \\
\text { uniquely impacted } \\
\text { by the pandemic } \\
\text { (ie, surgeons) }\end{array}$ \\
\hline $\begin{array}{l}\text { My Osler } \\
\text { iTunes: https://apps.apple.com/ } \\
\text { la/app/my-osler/id1504015523 } \\
\text { Google Play: https://play. } \\
\text { google.com/store/apps/ } \\
\text { details?id=com.oslertechnol- } \\
\text { ogy.myosler\&hl=en_US\&gl=US }\end{array}$ & $\begin{array}{l}\text { Medical education } \\
\text { and training (e-learn- } \\
\text { ing and teaching) }\end{array}$ & Free & $\begin{array}{l}\text { iTunes and } \\
\text { Google Play }\end{array}$ & $\begin{array}{l}\text { Scientific } \\
\text { journals }\end{array}$ & $\begin{array}{l}\text { Modules on PPE } \\
\text { and antiviral treat- } \\
\text { ments }\end{array}$ \\
\hline
\end{tabular}


TABLE Recommended COVID-19 apps (continued)

\begin{tabular}{|c|c|c|c|c|c|}
\hline App & Comprehensiveness & Price & Platform & $\begin{array}{l}\text { Literature } \\
\text { used }\end{array}$ & $\begin{array}{c}\text { Important special } \\
\text { features }\end{array}$ \\
\hline $\begin{array}{l}\text { unbound } \\
\text { Relief Central } \\
\text { iTunes: https://apps.apple.com/ } \\
\text { us/app/relief-central-covid-19/ } \\
\text { id353219185 }\end{array}$ & $\begin{array}{l}\text { - Clinical decision mak- } \\
\text { ing (clinical treatment } \\
\text { guidelines) } \\
\text { - Reference and } \\
\text { information gathering } \\
\text { (medical literature) }\end{array}$ & Free & iTunes & $\begin{array}{l}\text { CDC, WHO, } \\
\text { scientific } \\
\text { journals }\end{array}$ & $\begin{array}{l}\text { - Guidelines for } \\
\text { treatment, speci- } \\
\text { men collection } \\
\text { - Updates on new } \\
\text { coronavirus strains } \\
\text { - Vaccine guidelines } \\
\text { and effectiveness } \\
\text { studies }\end{array}$ \\
\hline $\begin{array}{l}\text { WHOA - WHO Academy (WHO) } \\
\text { iTunes: https://apps.apple.com/ } \\
\text { us/app/who-academy-covid19- } \\
\text { learning/id1506019873 } \\
\text { Google Play: https://play.google. } \\
\text { com/store/apps/details?id=org. } \\
\text { who.WHOA\&hl=en_US\&gl=US }\end{array}$ & $\begin{array}{l}\text { - Clinical decision mak- } \\
\text { ing (clinical treatment } \\
\text { guidelines) } \\
\text { - Reference and } \\
\text { information gathering } \\
\text { (medical literature) }\end{array}$ & Free & $\begin{array}{l}\text { iTunes and } \\
\text { Google Play }\end{array}$ & $\begin{array}{l}\text { WHO, scien- } \\
\text { tific journals }\end{array}$ & $\begin{array}{l}\text { - Modules on } \\
\text { pregnancy, breast } \\
\text { feeding } \\
\text { - Virtual learning } \\
\text { events (webinars) } \\
\text { - Targeted guides for } \\
\text { patients and health } \\
\text { care providers }\end{array}$ \\
\hline
\end{tabular}

Abbreviations: CDC, Centers for Disease Control and Prevention; COVID-19, coronavirus disease 2019; PAHO; Pan American Health Organization; PPE, personal protective equipment; UN, United Nations; WHO, World Health Organization.

References

1. World Health Organization. WHO coronavirus (COVID-19) dashboard. https://covid19.who.int/. Accessed March 12, 2021.

2. Kondylakis H, Katehakis DG, Kouroubali A, et al. COVID-19 mobile apps: a systematic review of the literature. $J$ Med Internet Res. 2020;22:e23170.
3. Bogaert K, Chen KT. COVID-19 apps for the ObGyn health care provider. OBG Manag. 2020; 32(5):44, 46.

4. Chyjek K, Farag S, Chen KT. Rating pregnancy wheel applications using the APPLICATIONS scoring system. Obstet Gynecol. 2015;125:1478-1483. 